\title{
MANNER OF DEATH OF OLDER PEOPLE WITH REGARD TO BLOOD ALCOHOL CONCENTRATION
}

\author{
Lubica Juríčková1, Kateřina Ivanová1, Martin Dobiáš² ${ }^{2}$ Richard Andrášik ${ }^{3}$, Peter Ondra² \\ ${ }^{1}$ Department of Social Medicine and Public Health, Faculty of Medicine and Dentistry, Palacký University Olomouc, Olomouc, Czech Republic \\ 2Department of Forensic Medicine and Medical Law, Faculty of Medicine and Dentistry, Palacký University Olomouc, Olomouc, Czech Republic \\ ${ }^{3}$ Department of Mathematical Analysis and Applications of Mathematics, Faculty of Science, Palacký University Olomouc, Olomouc, Czech Republic
}

\section{SUMMARY}

Objective: Alcohol abuse is related to a wide variety of negative health outcomes including mortality in older people. Alcohol abuse in older people is characterised by certain specific features uncommon in general adult population. The main objective of this study was to analyse the autopsy protocols of deceased older people in relation to blood alcohol concentration (BAC), sex, age, and manner of death. As a positive BAC, $>0.20 \mathrm{~g} / \mathrm{kg}$ was accepted.

Methods: The sample consists of 1,012 deceased older people (i.e. aged 65 years and over) selected out of 2,377 autopsied subjects in the period from 2003-2013. Subjects included into the sample were chosen via the proportional sampling method. Data (BAC, sex, age, and manner of death) was recorded in a single structured protocol. Data was evaluated statistically (Kolmogorov-Smirnov two-sample test, Wilcoxon two-sample test, risk ratio).

Results: Among older people, there has been a statistically significant correlation of natural death with sex (men died earlier) and with increased BAC (people with positive BAC died earlier). In case of violent death there is a difference in the types of accidents in older people with positive BAC $(>0.2 \mathrm{~g} / \mathrm{kg})$ and with negative BAC $(\leq 0.2 \mathrm{~g} / \mathrm{kg})$. Drowning is more common in older people with positive BAC.

Conclusions: Health campaigns in Europe and the Czech Republic aimed at reducing alcohol consumption mainly deal with young people. Alcohol abuse has an impact on premature mortality even in older people. As shown by this study, older people with positive BAC die significantly earlier.

Key words: older people, alcohol abuse, death, autopsy

Address for correspondence: K. Ivanová, Department of Social Medicine and Public Health, Faculty of Medicine and Dentistry, Palacký University Olomouc, Hněvotínská 3, 77515 Olomouc, Czech Republic. E-mail: katerina.ivanova@upol.cz

https://doi.org/10.21101/cejph.a4749

\section{INTRODUCTION}

The issue of alcohol abuse in older people (i.e. aged 65 years and over) is gaining societal importance, not only due to the rapidly growing proportion of these people in the population (1). Alcohol abuse in older people has some specific features compared to the general adult population. These specific features stem primarily from the differences in the physiological ageing of an organism, frequent polymorbidity in older people, usage of prescribed drugs and from different clinical picture of selected diagnostic units related to alcohol (2). Experts from the Centre for Ageing Research and Development in Ireland emphasize: "Many public health campaigns on the misuse of alcohol are aimed at younger age groups. However, there is evidence that alcohol misuse is increasing in people over the age of 65 (3)."

Reasons for alcohol abuse among older people are discussed in many studies (4-6). Another study explored whether individuals beginning harmful drinking after the age of 45 differ from alcoholics with an early onset (prior to the age of 25) (7). Alcohol abuse is not frequently diagnosed in older people (8). Some studies showed that alcohol abuse contributes to death and disability through accidents, injuries and suicide in older people (9-14). "The protection of health of populations by preventing and reducing the harmful use of alcohol is a public health priority, and one of the objectives of the World Health Organization is to reduce the health and social burden caused by the harmful use of alcohol" (15). Government priority is to reduce the potential risks associated with the use of alcohol and other drugs in the Czech Republic (CR) (1). One of the ways to determine the alcohol abuse related risks to health and life is to analyse the data of deceased people. Comprehensive data on deaths in older people may be found in autopsy protocols.

The aim of our survey was to analyse autopsy reports of deceased people recorded from 2003 to 2013 in relation to blood alcohol concentration (BAC), sex, age, manner of death, and other variables. This paper presents the manner of death of older people with regard to BAC.

\section{MATERIALS AND METHODS}

\section{Hypotheses}

The main null hypothesis postulated no statistically significant differences in the age structure and manner of death between men and women (aged 65 years and over) divided according to BAC. 
The main hypothesis was divided into 9 sub-hypotheses. Firstly, the data file of older people was divided into two groups according to sex (women and men) and according to BAC (positive BAC and negative BAC). Then, a comparison of the age structure and the manner of death was performed in both divisions. Afterwards, the data file of older people was divided into four groups according to sex and BAC simultaneously: women with positive $\mathrm{BAC}$; women with negative $\mathrm{BAC}$; men with positive $\mathrm{BAC}$; men with negative BAC. The objective of statistical analysis was to determine whether there are differences in the age distribution or manner of death in these four groups.

\section{Data Source}

The design was the retrospective forensic autopsy study. Autopsy reports from the years 2003-2013 were analysed at the Department of Forensic Medicine and Medical Law, Faculty of Medicine and Dentistry, Palacký University Olomouc, in 2014. The catchment areas of this site are two regions (Olomouc and Zlín, with about 1.2 million of inhabitants residing in both regions). The headquarters of the stated site approved the implementation of the survey.

\section{Sample and Sampling Strategy}

Proportional random sampling was used to choose $15 \%$ of autopsy reports per year out of the 15,941 autopsied people who died in the period from 2003 to 2013 (i.e. 2,377 autopsy reports from 2003-2013). It is a representative sample. Of the 2,377 files of deceased people, 1,012 people were aged 65 years and over 416 women (41.1\%) and 596 men (58.9\%).

\section{Research Tool and Procedure}

Required information from each autopsy report was manually recorded in a separate protocol. A structured protocol was specially created just for this survey. There were 14 variables of interest. These variables were consulted with experts in forensic medicine. Some variables, such as education, marital status, employment etc., could not be statistically evaluated because this information was not provided in the autopsy protocol. The following variables showed a significant statistical difference: age, sex, BAC, and manner of death. BAC was classified as negative $(\leq 0.20 \mathrm{~g} / \mathrm{kg})$ or positive $(>0.20 \mathrm{~g} / \mathrm{kg})(16)$. Almost all deceased people $(95 \%)$ were tested for the presence of alcohol. Tests for alcohol (ethanol) were carried out by gas chromatography. The validity of the data was checked in cooperation with two independent experts.

\section{Statistical Analysis}

The analysis of difference in age between the two groups was based on the Kolmogorov-Smirnov two-sample test and the Wilcoxon two-sample test (17). The standard level of significance of the test was chosen $(\alpha=0.05)$. Relative risk analysis was used to find differences in the type of accidents between the two groups (18). The relative risk analysis was also used for comparison of natural and violent death in both groups. The statistical analysis was performed in software R (19).

\section{Strengths and Limitations}

This is the most comprehensive survey of deceased older people with positive BAC in Olomouc and Zlín regions. This survey suffers mainly from the following limitations: not all information provided in the autopsy protocols of deceased older people was collected, e.g. deaths associated with the use of illicit drugs, pharmaceuticals etc.; only the data for the 2003-2013 period was analysed.

\section{RESULTS}

\section{Characteristics of Deceased Older People}

Table 1 states the age, BAC and manner of death of older people (i.e. aged 65 years and over) in 2010-2013. Median age of men was significantly lower than the median age of women (Wilcoxon two-sample test, p-value $<0.0001$ ). The data show a statistically significant relationship of alcohol influence and sex. The positive BAC $(>0.2 \mathrm{~g} / \mathrm{kg})$ was 2.63 times more common in men than in women $-95 \%$ confidence interval of risk ratio (RR) was 1.75-3.94. It was also demonstrated that the mean age of older people with positive BAC was significantly lower than the mean age of older people with negative BAC (Wilcoxon twosample test, p-value $<0.0001)$. Of the 1,012 autopsied individuals, natural death was reported in 787 cases $(77.8 \%)$, violent death in 213 cases $(21.0 \%)$.

Figure 1 shows percentages of deceased older people according to sex, BAC and manner of death. Percentages were calculated with respect to the manner of death. It means that for each manner of death (natural death, accident, suicide, and homicide) the

Table 1. Age, blood alcohol concentration (BAC) and manner of death in older people $(N=1,012,65+)$

\begin{tabular}{|c|c|c|}
\hline & Women $(n=416)$ & $\operatorname{Men}(n=596)$ \\
\hline \multicolumn{3}{|l|}{ Age } \\
\hline Minimum & 65.0 & 65.0 \\
\hline Median & 78.0 & 74.0 \\
\hline Mean & 78.8 & 74.9 \\
\hline Maximum & 99.0 & 97.0 \\
\hline Standard deviation & 7.6 & 7.3 \\
\hline \multicolumn{3}{|c|}{ Blood alcohol concentration } \\
\hline$>0.2 \mathrm{~g} / \mathrm{kg}$ (positive) & $27(2.7 \%)$ & $101(10.0 \%)$ \\
\hline$\leq 0.2 \mathrm{~g} / \mathrm{kg}$ (negative) & $371(36.7 \%)$ & $466(46.0 \%)$ \\
\hline Undetermined & $18(1.8 \%)$ & $29(2.9 \%)$ \\
\hline \multicolumn{3}{|l|}{ Manner of death } \\
\hline Natural death & $331(32.7 \%)$ & $456(45.1 \%)$ \\
\hline Violent death & $79(7.8 \%)$ & $134(13.2 \%)$ \\
\hline Accident & $41(4.1 \%)$ & $68(6.7 \%)$ \\
\hline Suicide & $23(2.3 \%)$ & $50(4.9 \%)$ \\
\hline Homicide & $2(0.2 \%)$ & $1(0.1 \%)$ \\
\hline Other & $13(1.3 \%)$ & $15(1.5 \%)$ \\
\hline Undetermined & $6(0.6 \%)$ & $6(0.6 \%)$ \\
\hline
\end{tabular}


percentages sum up to $100 \%$. Regarding natural death, there were $41.9 \%$ of women, $38.0 \%$ of women were among people who died as a result of an accident. Concerning suicides, there were $33.3 \%$ of women and two women out of 3 people who died as a result of a homicide.

We analysed relative frequencies displayed in Fig. 1 by the use of RR. Natural death in men with positive BAC was relatively more common than in women with positive BAC. More specifically, natural death in men with positive BAC was by 4.26 more frequent than natural death in women with positive BAC, with $95 \%$ confidence interval of RR accounting for 2.21-8.20. Similarly, death due to an accident in men with positive $\mathrm{BAC}$ was relatively more frequent (3.52 times) than in women with positive $\mathrm{BAC}-95 \%$ confidence interval of RR was 1.32-9.41. The proportion of men with positive $\mathrm{BAC}$ was twice as high as the proportion of women with positive BAC $-95 \%$ confidence interval of RR was $1.63-6.39$. The number of records related to homicide was very low (only three). Therefore, we have not analysed this group of older people separately.

\section{Natural Death}

Fig. 2 shows that men aged 65 years and over die earlier of natural death than women aged 65 years and over $(<0.0001)$.

Fig. 3 compars deceased older people with positive $\mathrm{BAC}(>0.2$ $\mathrm{g} / \mathrm{kg})$ to deceased older people with negative BAC $(\leq 0.2 \mathrm{~g} / \mathrm{kg})$. It shows that older people with positive BAC die earlier than older people with negative BAC $(<0.0001)$.

\section{Violent Death}

Table 2 shows the most interesting findings in the analysis of violent deaths - drowning. It is significantly more common than other types of accidents in older people with positive BAC compared to older people with negative BAC. When compared to other group pairs (e.g. women and men, BAC positive and BAC negative), the data does not suggest any significant difference in the type of accident. There were only few cases of suicides and homicides in the data file. Therefore, we have not found out any significant conclusions for these two categories.

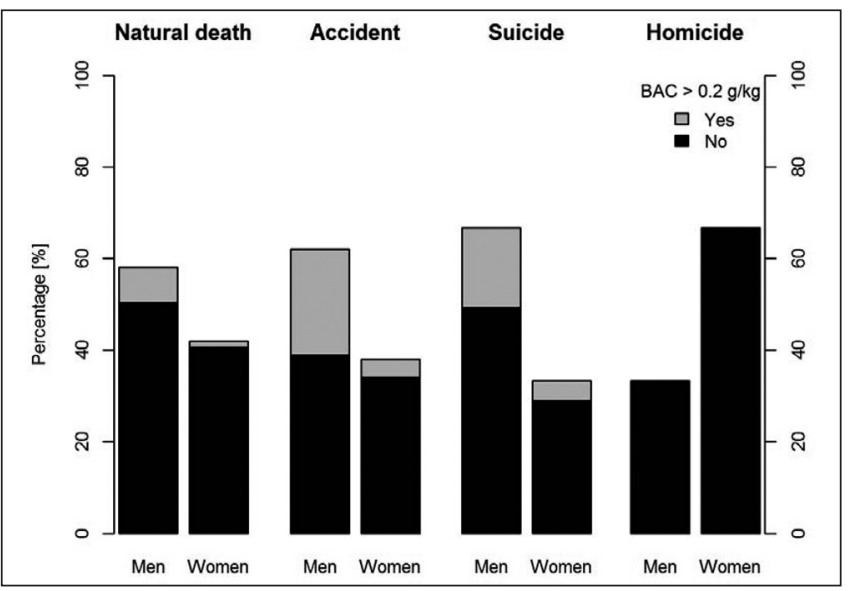

Fig. 1. Percentage of deceased older people according to sex, blood alcohol concentration (BAC) and manner of death.

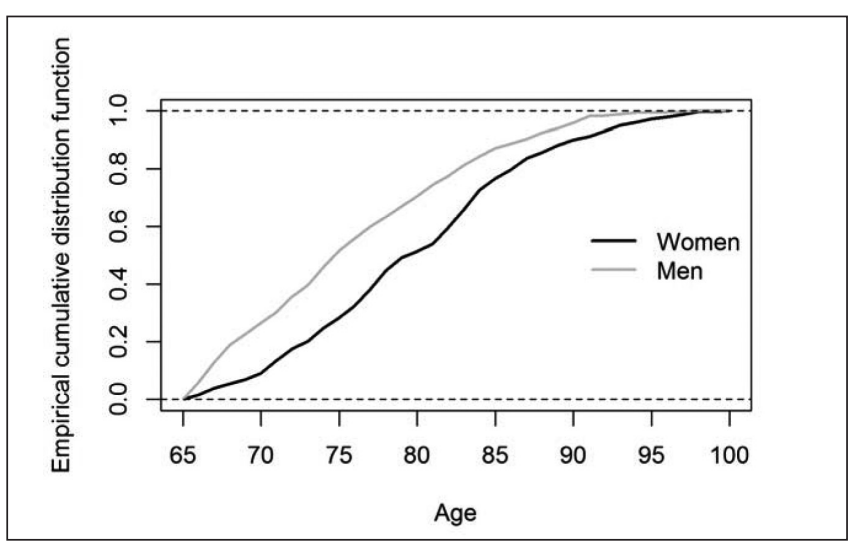

Fig. 2. Age distribution of older people who died of natural death.

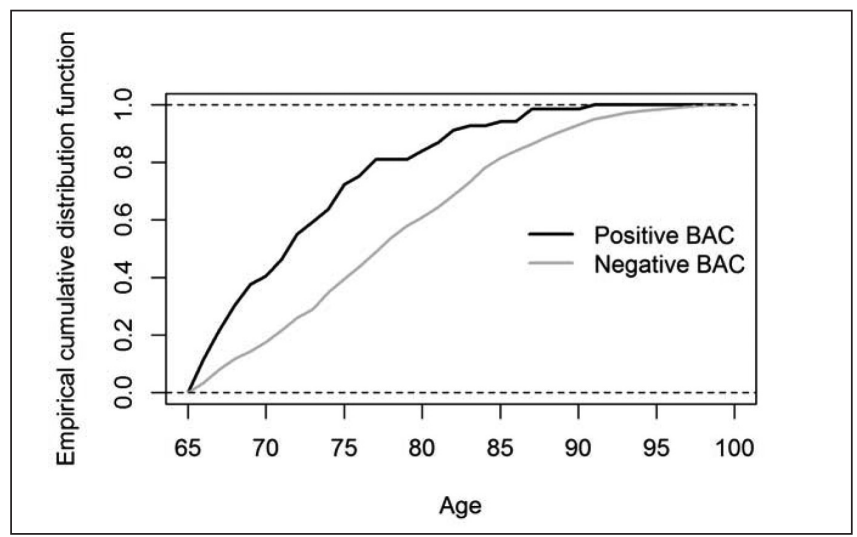

Fig. 3. Age distribution of older people with blood alcohol concentration (BAC) who died of natural death.

\section{Comparison of Natural and Violent Death}

Table 3 shows the results of testing of the differences between natural and violent death in older people in relation to sex and BAC (positive and negative). It has been found out that violent death was 2.48 times more frequent in older people with positive BAC than in older people with negative BAC. Violent death was 3.47 times (and 2.20 times) more frequent in women and men, respectively, aged 65 years and over with positive BAC than in women and men, respectively, aged 65 years and over with negative BAC. No statistically significant difference was identified in the other compared groups.

\section{DISCUSSION AND CONCLUSIONS}

The results of this retrospective study (2003-2013) show a statistically significant dependence of natural death on sex (men die earlier) and positive BAC (people with positive BAC die earlier) in older people. In violent death, there is a difference in the type of accident between individuals with positive $\mathrm{BAC}(>0.2$ $\mathrm{g} / \mathrm{kg})$ and with negative BAC $(\leq 0.2 \mathrm{~g} / \mathrm{kg})$. Drowning is more common in older people with positive BAC. There is a statistically significant difference in groups of people with positive and negative BAC when comparing the natural and violent death. Among women, this difference is higher than among men. It is, therefore, possible to conclude that alcohol abuse has an impact on premature mortality in older people. Compared with younger 
Table 2. Accident types by deceased older people with positive blood alcohol concentration (BAC) compared to older people with negative $B A C(N=1,012,65+)$

\begin{tabular}{|l|c|c|c|}
\hline Accident types & Risk Ratio & $95 \% \mathrm{Cl}$ & Statistically significant difference \\
\hline Drowning & 3.24 & $1.08-9.76$ & Yes \\
\hline Falls & 0.58 & $0.27-1.24$ & No \\
\hline Poisoning & 2.16 & $0.63-7.46$ & No \\
\hline Vehicular incident & 0.80 & $0.33-1.94$ & No \\
\hline Others & 0.90 & $0.40-2.03$ & No \\
\hline
\end{tabular}

Table 3. Comparison of natural and violent death in older people in relation to sex and positive and/or negative blood alcohol concentration $(B A C)(N=1,012,65+)$

\begin{tabular}{|l|c|c|c|}
\hline Groups & Risk Ratio & $95 \% \mathrm{Cl}$ & $\begin{array}{c}\text { Statistically significant } \\
\text { difference }\end{array}$ \\
\hline Women total vs. Men total & 0.85 & $0.66-1.09$ & No \\
\hline Positive BAC total vs. Negative BAC total & 2.48 & $1.93-3.18$ & Yes \\
\hline Positive BAC women vs. Negative BAC women & 3.47 & $2.31-5.22$ & Yes \\
\hline Positive BAC men vs. Negative BAC men & 2.20 & $1.61-3.00$ & Yes \\
\hline Negative BAC women vs. Negative BAC men & 0.93 & $0.69-1.25$ & No \\
\hline Positive BAC women vs. Positive BAC men & 1.47 & $0.97-2.22$ & No \\
\hline
\end{tabular}

and middle-aged population, alcohol consumption affects more men than women even in older people.

An analysis of the entire study population of deceased people $(2,377)$ in 2003 and 2013 showed a statistical dependence between alcohol test positivity and sex (more often in men), age (more common in younger individuals) and the manner of death (violent death more common) in both analysed years (20). There was also a significant correlation between alcohol test positivity, sex and manner of death in the group of older people $(1,012)$ compared to the entire study population $(2,377)$.

A number of authors focused on the issue of alcohol abuse in deceased individuals in relation to their BAC (21-24). Our retrospective autopsy study (2003-2013) differs mainly by examining not only the sex, age and violent death, but also natural death cases associated with positive and negative BAC in older people. Some authors address one specific subgroup of violent death causes, namely e.g. traffic accidents or suicide, or they focus on different socio-demographic characteristics among deceased people (10, $13,14)$. In another study general practitioners declare strong support and demand to implement early intervention programmes aimed at alcohol problems (25).

In 2015, the Ministry of Health of the Czech Republic issued the action plan to limit the harm caused by alcohol. It is the first document that contains complex design of activities in order to reduce the alarmingly high alcohol consumption in the population of the CR and related negative societal impacts, including both the negative health impact on individuals' health as well as social and economic consequences. The alcohol issue has not been managed in any coordinated way so far, although it is a major public health problem in the CR. The main goal of this action plan is a fundamental change in the highly tolerant attitude of the Czech population towards alcohol use and the associated reduction in alcohol consumption to limit the harm caused by its use. High alcohol consumption with considerable negative societal impacts has financial implications for the state budget (1). Another study reported growing expenditures of healthcare systems all over the world, including the CR. One of the basic factors of expenditure growth is the ageing of population (26). Important components in active ageing are personal active ageing strategies of older people, which include lower alcohol consumption (27). Our study shows the combination of alcohol consumption with injury prevention in older people. The results might serve to improve the plan for the lowering of alcohol consumption among older people as well.

\section{Conflict of Interests}

None declared

\section{Acknowledgements}

This study was supported by Palacký University Olomouc (Grant No IGA_LF_2014_033) and RVO 61989592.

\section{REFFERENCES}

1. Ministry of Health of the Czech Republic. Health 2020 - Action Plan no. 4c [Internet]. Prague: Ministry of Health of the CR; 2015 [cited 2015 Dec 10]. Available from: http://www.mzcr.cz/verejne/dokumenty/akcniplany-pro-implementaci-narodni-strategie-zdravi-2020_10814_3016_5. html. (In Czech.)

2. Skopalová B. The Influence of Alcohol on the Health Status of Seniors Adiktologie. 2014 July;14(4):420-7. (In Czech.)

3. Centre for Ageing Research and Development. Focus on alcohol misuse among older people aged 65 or over [Internet]. Ireland: CARDI; 2013 [cited 2015 Jan 10]. Available from: http://www.drugs.ie/resourcesfiles/ ResearchDocs/Ireland/2013/Focus_on_alcohol_misuse_among_older_ people $02 \quad 8 \quad 13$ jh.pdf.

4. Atkinson PM. Aging and alcohol use disorders: diagnostic issues in the elderly. International Psychogeriatrics. 1990 Spring;2(1):55-72.

5. Dar K. Alcohol use disorders in older people: fact or fiction? Advances in Psychiatric Treatment. 2006 July;12(3):173-81.

6. Fink A, Hays RD, Moore AA, Beck JC. Alcohol-Related Problems in Older Persons. Arch Intern Med. 1996 Jun;156(11):1150-6. 
7. Wetterling T, Veltrup C, John U, Driessen M. Late onset alcoholism. European Psychiatry. 2003 May;18(3):112-8.

8. Curtis JR, Geller G, Stokes EJ, Levine DM, Moore RD. Characteristics, diagnosis, and treatment of alcoholism in elderly patients. J Am Geriatr Soc 1989Apr;37(4):310-6.

9. Zaridze D, Maximovitch D, Lazarev A, Igitov V, Boroda A, Boreham J, et al. Alcohol poisoning is a main determinant of recent mortality trends in Russia: evidence from a detailed analysis of mortality statistics and autopsies. Int J Epidemiol. 2009 Feb;38(1):143-53.

10. Jones WA, Kugelberg FC, Holmgren A, Ahlner J. Five-year update on the occurrence of alcohol and other drugs in blood samples from drivers killed in road-traffic crashes in Sweden. Forensic Science International. 2009 Apr;186(1-3):56-62.

11. Sorock GS, Chen LH, Gonzalgo SR, Baker SP. Alcohol-drinking history and fatal injury in older adults. Alcohol. 2006 Nov;40(3):193-9.

12. Grundstrom AC, Guse CE, Layde PM. Risk factors for falls and fallrelated injuries in adults 85 years of age and older. Arch Gerontol Geriatr. 2012 May-Jun;54(3):421-8.

13. Kaplan MS, McFarland BH, Huguet N, Conner K, Caetano R, Giesbrecht $\mathrm{N}$, Nolte KB. Acute alcohol intoxication and suicide: a gender-stratified analysis of the National Violent Death Reporting System. Injury Prevention. $2013 \mathrm{Feb} ; 19(1): 38-43$.

14. Caetano R, Kaplan MS, Huguet N, McFarland BH, Conner K, Giesbrecht $\mathrm{N}$, Nolte KB. Acute Alcohol Intoxication and Suicide among United States Ethnic/Racial Groups: Findings from the National Violent Death Reporting System. Alcoholism: Clinical and Experimental Research. 2013 May;37(5):839-46.

15. World Health Organization. Global status report on alcohol and health 2014. Geneva: WHO; 2014

16. Bulletin of the Ministry of Health of the Czech Republic. 2006 Sep;(7):15. (In Czech.)

17. Corder GW, Foreman DI. Nonparametric Statistics for Non-Statistians: A Step-by-Step Approach. New Jersey (USA): Wiley; 2009.

18. Simon SD. Understanding the Odds Ratio and the Relative Risk. Journal of Andrology. 2001 July-Aug;22(4):533-6.
19. R Development Core Team. R: A language and environment for statistical computing. R Foundation for Statistical Computing. Vienna (Austria); 2008.

20. Juríčková L, Dobiáš M, Ivanová K, Ondra P. Problems of alcohol abuse at deceased people in the Olomouc and Zlín Regions: a pilot study. Gnosis Medica. 2014;2(2):16-22. (In Czech.)

21. Straka L, Zubor P, Novomesky F, Stuller F, Krajcovic J, Kajo K, Danko J. Fatal alcohol intoxication in woman: A forensic autopsy study from Slovakia. BMC Public Health. 2011 Dec;11:924.

22. Holmgren A, Jones AW. Demographics of suicide victims in Sweden in relation to their blood-alcohol concentration and the circumstances and manner of death. Forensic Science International. 2010 May;198(1-3):1722.

23. Lunetta P, Pentillä A, Sarna S. The Role of Alcohol in Accident and Violent Deaths in Finland. Alcoholism: Clinical and Experimental Research. 2001 Nov;25(11):1654-61.

24. Darke S, Duflou J, Torok M. Drugs and violent death: comparative toxicology of homicide and non-substance toxicity suicide victims. Addiction. 2009 Jun;104(6):1000-5.

25. Anderson P, Wojnar M, Jakubczyk A, Gual A, Reynolds J, Segura L, et al. Managing Alcohol Problems in General Practice in Europe: Results from the European ODHIN Survey of General Practitioners. Alcohol and Alcoholism. 2014 July;49(5):531-9.

26. Zelený T, Bencko V. Healthcare system financing and profits: all that glitters is not gold. Cent Eur J Public Health. 2015 Mar;23(1):3-7.

27. Klugar M, Č́p J, Klugarová J, Marečková J, Roberson DN, Kelnarová Z. The active aging personal strategies of older adults in Europe: a systematic review of qualitative evidence. JBI Database System Rev Implement Rep. 2016 Aug;14(5):193-257.

Received February 29, 2016 Accepted in revised form February 2, 2017 